\title{
Developmental changes in story-evoked responses in the neocortex and hippocampus
}

\author{
Samantha S. Cohen ${ }^{1}$ and Christopher Baldassano ${ }^{1}$ \\ ${ }^{1}$ Columbia University, Department of Psychology
}

\begin{abstract}
How does the representation of naturalistic life events change with age? Here we analyzed fMRI data from 415 children and adolescents (5 - 19 years) as they watched a narrative movie. In addition to changes in the degree of inter-subject correlation (ISC) with age in sensory and medial parietal regions, we used a novel measure (between-groups ISC) to reveal age-related shifts in the responses across the majority of the neocortex. Over the course of development, brain responses became more discretized into stable and coherent events, and shifted earlier in time to anticipate upcoming event transitions. However, hippocampal responses to event boundaries actually decreased with age, suggesting a shifting division of labor between episodic encoding processes and schematic event representations between the ages of 5 and 19 .
\end{abstract}




\section{Introduction}

The ability to perceive and remember the world changes radically throughout the first twenty years of life (Aslin, \& Smith, 1988; Schneider, \& Pressley, 2013). Older children and adolescents are better able to understand and interpret the world around them, and anticipate upcoming situations (Carpendale, and Lewis, 2006; Casillas, \& Frank, 2017; Richardson \& Saxe, 2020). Much of this increase in ability can be attributed to greater familiarity with the events that they are likely to encounter (Schneider, \& Pressley, 2013). For instance, children have better memory for situations that they have expertise in (e.g. Chess; Chi, 1978). Here, we characterize changes that occur in the brain's response to complex naturalistic stimuli during this period of the acquisition of structured knowledge about the world.

Previous research has examined developmental changes in neural activity when learning principled relationships between items in a laboratory setting (Brod, Lindenberger, \& Shing; 2017). Children, however, normally acquire and organize novel information about their world over the course of weeks, if not years (Nelson, 1986). Naturalistic narrative stimuli provide a tool for probing this complex, real-world knowledge that children have acquired across repeated experiences and can deploy automatically (Cantlon and Li, 2013; Lerner et al., 2019; Petroni et al., 2018; Moraczewski et al., 2018; 2020; Richardson et al., 2018; Richardson \& Saxe, 2020; Vanderwal et al, 2019).

We utilized functional magnetic resonance imaging (fMRI) data acquired while children and adolescents between the ages of 5 and 19 watched a short narrative cartoon that contained both social and emotional content (Alexander et al., 2017; Petroni et al., 2018). Although cartoons do not physically replicate the characteristics of everyday perception, there is evidence to suggest that children respond similarly to the social cues in cartoons and live action videos (Han, Jiang, \& Humphreys, 2007). Analyzing brain responses to complex stories or movies is challenging, since we do not yet have models that can predict brain-wide responses to these types of stimuli, especially given the fact that the representation of meaning likely changes with age (Clark, 1973). A model-free approach to assess brain responses to this kind of stimuli is inter-subject correlation (ISC), which measures the similarity of brain responses in a brain region across movie viewers (Cantlon and Li, 2013; Lerner et al., 2019; Petroni et al., 2018; Moraczewski et al., 2018; 2020). ISC within an age group has generally been found to increase with age, possibly due to more mature engagement with the content, greater shared knowledge about the world, or more exposure to widely used cinematic conventions (Cantlon and Li, 2013; Lerner et al., 2019; Moraczewski et al., 2018; 2020). We therefore measured ISC in parcels throughout the cortex (Schaefer et al., 2018) to assess if processing becomes more similar with age. In line with previous studies, we hypothesized that the magnitude of ISC would increase with age, although decreases have been found in studies in other modalities (Petroni et al., 2018).

ISC has also been measured between age groups to assess the similarity between the responses of children and adults (Cantlon and Li, 2013; Lerner et al., 2019; Moraczewski et al., 2018). If a child is more similar to adults, they are considered more mature, and therefore likely to have better academic or social abilities (Cantlon and Li, 2013; Moraczewski et al., 2018). This previous work is consistent with the idea that children are simply noisy versions of adults, and the noise level decreases with age. We test an alternative hypothesis: that children and adolescents have different average response 
timecourses, corresponding to different age-related interpretations of the movie. We therefore employed a new across-group ISC measure that allows for comparisons across age groups while controlling for the degree of similarity within each age group. This allowed us to determine whether there was a consistent shift in the group-level temporal patterns of activity, independent of changes in within-group consistency. We hypothesized that in many brain regions, even those where within-age ISC magnitude does not change, the pattern of activity representing the movie will change across development, just as the semantic interpretation of movies changes with age (Nelson, 1986).

How can we characterize the changes that are occurring in response timecourses? Recent work in adults has shown that the neural activity evoked by narratives are characterized by stable patterns of responses, and that the moments of transition between stable patterns in association areas correspond to meaningful boundaries between "events" in a continuous perceptual stream (Baldassano et al., 2017; 2018; Chen et al., 2017). These event patterns are driven in part by learned schematic scripts about common experiences in the world, such as the sequence of events that characterize a visit to a restaurant (Baldassano, Hasson, and Norman; 2018). Here, following this previous research in adults, we assessed the ability of children of different ages to segment their world into discrete chunks with a Hidden Markov model (HMM; Baldassano et al., 2017; 2018; Lee et al., 2021). We predicted that activity patterns will be more consistent among older subjects and become more stable across timepoints within events, providing a better fit to the HMM event segmentation model.

It is also likely that the timing of event transitions changes as a function of age. Activity in regions responsible for representing the viewpoints of others occurs earlier during the second presentation of a movie in children between six and seven years then in children between three and four years (Richardson and Saxe, 2020). Here we test whether, on an initial viewing of a naturalistic video, the timing of event transitions varies with age. Unlike previous work that considered anticipation at only a fixed offset of two seconds (Richardson and Saxe, 2020), the HMM-derived transition timings provide a flexible approach for detecting timing shifts between age groups that can vary across brain regions with different processing timescales (Baldassano et al., 2017; Hasson, Chen, \& Honey, 2015) and can vary across timepoints throughout the movie. We expected that older adolescents would be able to anticipate events further into the future due to their increased experience with the world.

Another characteristic of adult responses to narrative movies is the robust hippocampal activity evoked by boundaries between events (Baldassano et al., 2017; Ben-Yakov and Henson, 2018). Age-related changes in this signal have been previously observed in older adults, with response decreases between ages 18 and 88 in the posterior hippocampus (Reagh et al., 2020). It is therefore possible that development of event-structured responses in the cortex is mirrored by changes in the hippocampus's ability to respond to event transitions. We predicted that the magnitude of the hippocampal response to event boundaries would increase with age, in line with a model of maturation wherein expertise increases into middle age and then decreases with senescence (Grady, 2012; Reagh et al., 2020). 


\section{Results}

We sought to uncover whether and how the neural representation of naturalistic stimuli change with age between 5 and 19 years. To accomplish this, we used a large, publicly available dataset (Alexander et al., 2017) of functional magnetic resonance imaging (fMRI) data recorded while children watch a short video animation with both social and emotional themes. As the data were not equally distributed with age, the results are derived from equally-sized subsamples from each age group (each with an age span of approximately 3 years). To maximize the power and reproducibility of the results, our analyses were averaged across five random subsamples from each age group (Poldrack et al., 2017).

We first measured inter-subject correlation (ISC) across all subjects, as a general measure of story comprehension and engagement (Supplementary Figure 1; Nastase et al., 2019). To statistically examine changes in this measure due to age, we compared ISCs for the youngest (5-8 years) and oldest (16-19 years) age groups, calculated within parcels (Schaefer et al., 2018). We found (Figure 1) that ISC increases with age in low level sensory regions such as the auditory and visual cortex, and decreases with age in some higher-level association regions such as the posterior medial cortex (PMC), a region which includes both the posterior cingulate cortex (PCC) and retrosplenial cortex (RSC).

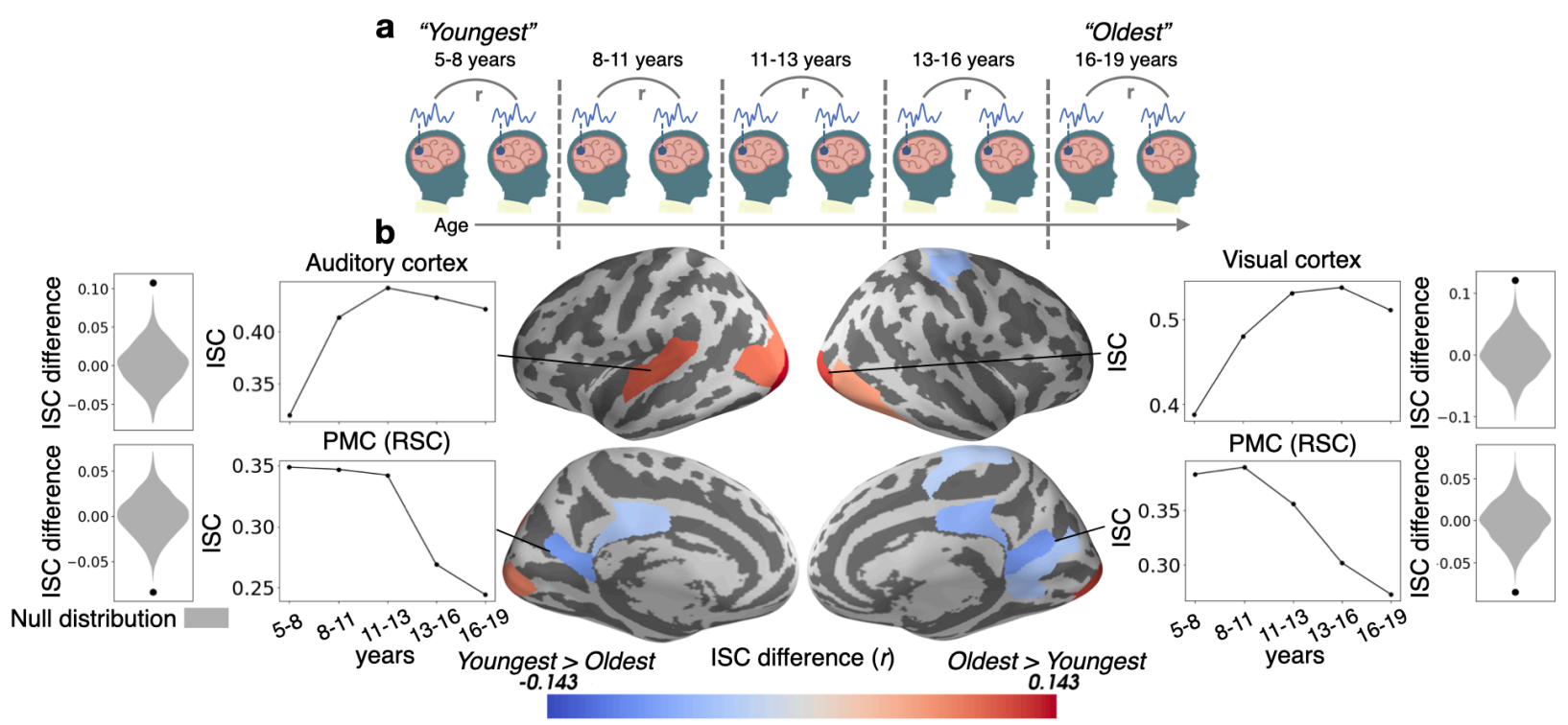

Figure 1: Inter-subject correlation (ISC) increases with age in sensory regions such as the auditory and visual cortices and decreases with age in the PMC. (a) Within-group ISC was computed for five distinct age groups, and a statistical comparison was performed between the Oldest and Youngest age groups. (b) The difference in ISC between the Youngest (5-8 years) group and the Oldest (16-19 years) group is displayed in significant parcels $(q<0.05)$ on the cortical surface. ISCs for all age groups are plotted for four significant parcels, selected post hoc for illustration, along with the ISC difference between the Youngest and Oldest groups compared to the null distribution.

In most of the cortex there is no difference in ISC magnitude as a function of age, which could indicate that children of all ages engage similarly with the movie (Cohen et al., 2018). It is also possible, however, that responses are highly consistent within each age group but 
differ systematically across age groups. For instance, it is possible that the most salient features of the video change with age, eliciting responses that are predictable within an age group but different across groups. To get a pure measure of across-group similarity that is insensitive to within-group variation, we use a novel measure called between-group ISC (ISC ${ }_{b}$; see Appendix A) (Figure 2a). To calculate the correlation between groups, ISCb is computed by dividing the between-group (Youngest to Oldest) correlation by the geometric mean of the within-group correlation (Figure $2 b$ ).

a

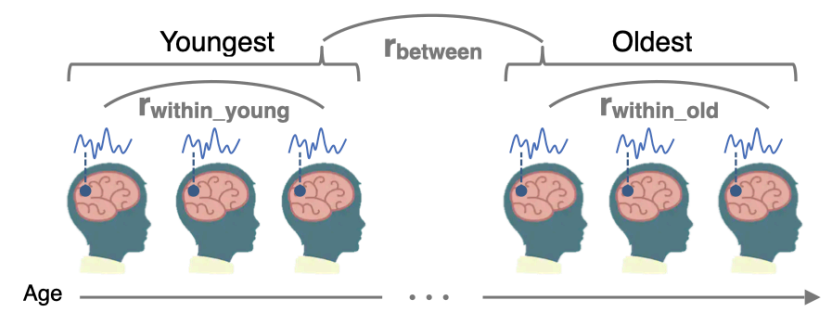

b

$$
I S C_{b}=\frac{r_{\text {between }}}{\sqrt{r_{\text {within_young }}} \sqrt{r_{\text {within_old }}}}
$$

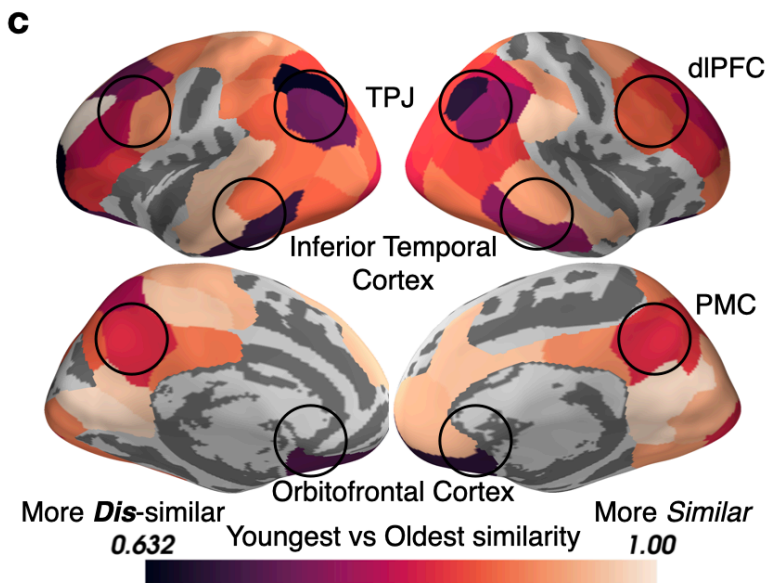

C

Figure 2: Stimulus processing differs in semantic and default mode regions between the Youngest and Oldest ages. (a) Response timecourses were correlated within the Oldest and Youngest groups separately, and also correlated between groups. (b) For each parcel, between-groups ISC ( $\mathrm{ISC}_{b}$ ) is calculated by dividing the across-group correlation by the geometric mean of the within-group correlation. This allows us to identify regions in which the correlations between groups are smaller than we would expect based on the within-group similarities, reflecting differing mean responses across groups. (c) The Youngest and Oldest groups are most dissimilar (indicated by darker colors) in regions in the default mode network, such as the temporoparietal junction, and in regions responsible for semantic processing, such as the inferior temporal cortices, temporoparietal junction, and dorsolateral prefrontal cortex (parcels shown have ISC $_{b}$ values less than age-shuffled null permutations, thresholded at $\mathrm{q}<0.05)$.

Eighty-one of the 100 Schaefer et al. (2018) parcels tested show significant age-specific response timecourses including default mode regions, such as the temporoparietal junction (TPJ) and PMC (Raichle et al., 2001), and language and concept sensitive regions, such as the dorsolateral prefrontal cortex (dlPFC) and inferior temporal cortices, and orbitofrontal cortex (Binder, \& Desai, 2011; Ralph, Jefferies, Patterson, \& Rogers, 2017; Figure 2c).

This result demonstrates that responses are changing substantially with age in many brain regions, but does not indicate how these responses are changing. One hypothesis is that the temporal dynamics of response patterns become more event-structured with development. In adults, movie responses are often characterized by rapid transitions between stable periods of activity, corresponding to meaningful events in the narrative (Baldassano et al., 2017; Chen et al., 2017). To identify the extent of this temporal clustering in our data, and the timescale of this clustering, we use a Hidden Markov Model (HMM) (Baldassano et al., 2017). Fitting the HMM separately to the Youngest and Oldest groups, we found that the HMM was able to identify event structure in at least one of the 
groups (Youngest and/or Oldest) for 70 out of our 100 parcels (Supplementary Figure 2). In these 70 parcels, we found that the optimal timescale (optimal number of events) was highly correlated between the Youngest and Oldest ages $(\mathrm{r}=0.87$, RMS difference between groups $=11.8+/-14.7$ seconds), showing increasing timescales from sensory regions to higher-level association regions (Supplementary Figure 3). This replicates previous research showing increasing event timescales across the cortical hierarchy (Baldassano et al., 2017; Geerligs, et al., 2021; Hasson, Chen, \& Honey, 2015), and shows that this timescale hierarchy is present even in our Youngest (5-8 years) age group.

As all 70 parcels had similar timescales in both groups, we jointly fit an HMM (with the parcel's optimal number of events) simultaneously to the Youngest and Oldest groups. As expected based on previous research in adults, the event boundaries form this model correspond to the boundaries subjectively placed by human raters in association regions, such as PMC, TPJ, and precuneus (Supplementary Figure 4; Baldassano et al., 2017). The jointly fit HMM produced an ordered set of event patterns which were shared across both groups (Figure 3a). We then tested this event model in held-out subjects, allowing us to measure in each group: (1) the model fit (log likelihood on held-out data), indicating the extent to which brain responses could be explained as an ordered sequence of these stable event patterns (Figure $3 \mathrm{~b}^{-c}$ ), and (2) the timing of event transitions. We hypothesized that the Oldest subjects would have a better model fit than the Youngest subjects, indicating increased stability and reliability of event representations. We also hypothesized that Oldest group would exhibit earlier event transitions than Youngest group, due to predictive, anticipatory, or preparatory processes (Carpendale and Lewis, 2006; Lee et al., 2021; Richardson \& Saxe, 2020).

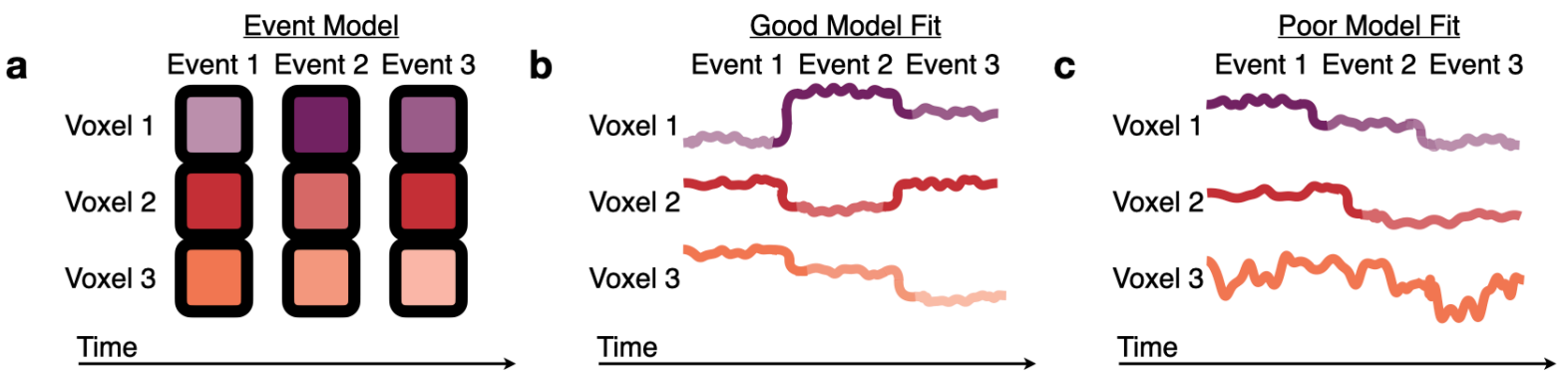

Figure 3: Measuring fits to the HMM event model. (a) The Hidden Markov Model (HMM) assumes that brain activity in response to a movie should proceed through a specific sequence of stable event patterns, each with a specific pattern of high and low activities across voxels (represented here as the saturation of each color). (b) The model is a good fit to brain responses that exhibit patterns consistent with the model assumptions, sequentially transitioning between the HMM event patterns with little variability during events. (c) A poor model fit indicates that this event model does not capture a brain region's dynamics, because the order of the relative activity levels does not match the model's sequence of event patterns (Voxel 1), the event transitions do not align between voxels (Voxel 2), or there is high within-event variability (across time or across subjects) (Voxel 3).

As hypothesized, the neural activity of the Oldest group can be better represented by an event model in most of the parcels that significantly differ with age. These regions include sensory areas, such as the auditory and visual cortex, as well as the left TPJ and dlPFC 
(Figure 4). In sensory regions, the largest change in event model-fit occurs between children $5-8$ years and those $8-11$ years (Figure 4 , side panels). The temporoparietal junction increases in model-fit through approximately age 13. These developmental trajectories hold not just for these regions' optimal event timescale, but also across a wide range of event timescales (Figure 4, side panels). Surprisingly, the HMM exhibits better model fits in the PMC for the Youngest group than the Oldest group. These results partially reflect changes in across-subject consistency as identified by the model-free ISC analysis (Figure 1), but also show effects in additional regions including the dlPFC and left TPJ.

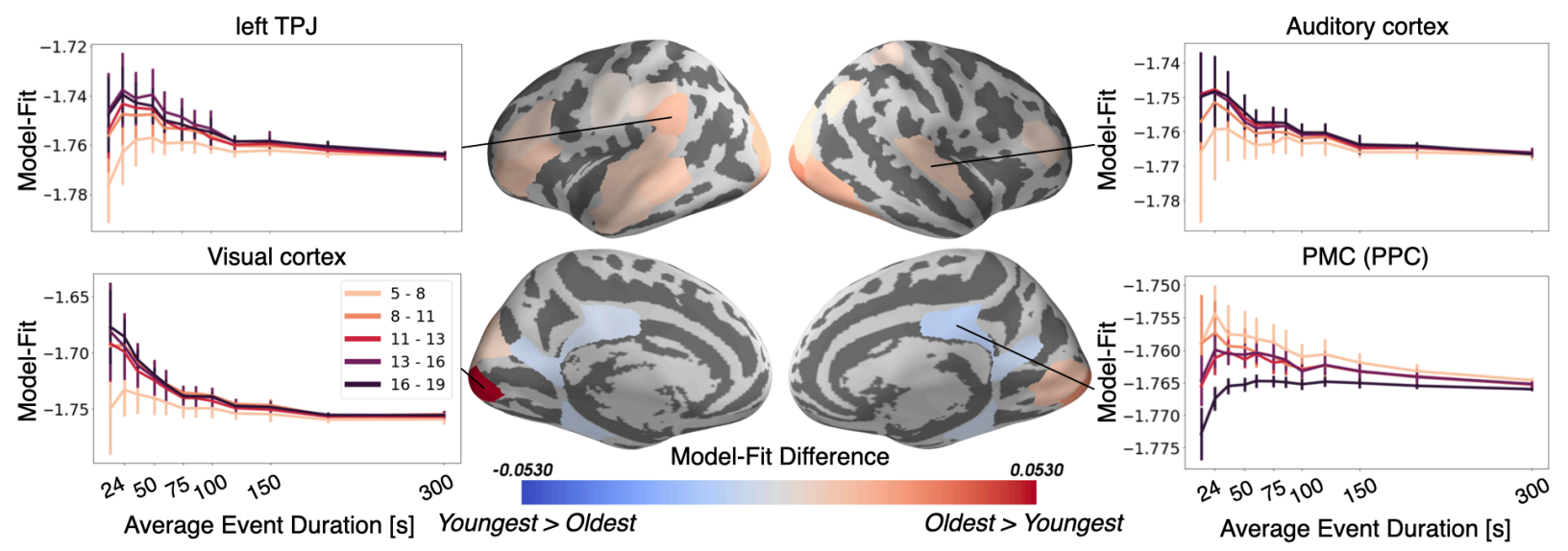

Figure 4: The Oldest group has better event models in regions including visual cortex, auditory cortex and temporoparietal junction, whereas the Youngest group has a better model-fit in PMC. Regions with a significant model-fit difference between the youngest and oldest age group (at the optimal timescale for that region) are plotted on the cortical surface. Redder shades indicate that the event model fits improve with age, and bluer shades indicate that they weaken with age. The model fits for event models with different event durations are shown across all age groups and event durations in four example regions, selected post hoc for illustration.

We also expected that event boundaries would occur earlier in time with age, because adolescents are more experienced with real-world schemas and can thus predictively represent upcoming situations. This hypothesis was borne out in sensory regions including auditory and visual cortex, as well as left lateralized associative regions including the left temporoparietal junction, left precuneus, and left dlPFC (Figure 5). Sensory regions are predictive at shorter time scales, consistent with their quicker processing speed (Baldassano et al., 2017; Hasson, Chen, \& Honey, 2015; Lee et al., 2021), while the higherlevel association regions anticipate upcoming events on average up to tens of seconds into the future. This anticipation increases rapidly across ages 5 to 11 in the left dlPFC and left precuneus, with a more variable trajectory in left TPJ (side panels). However, as was seen for model-fit, this trend reverses in the PMC where the Youngest children anticipate upcoming events slightly sooner, and anticipation decreases almost linearly with age. 


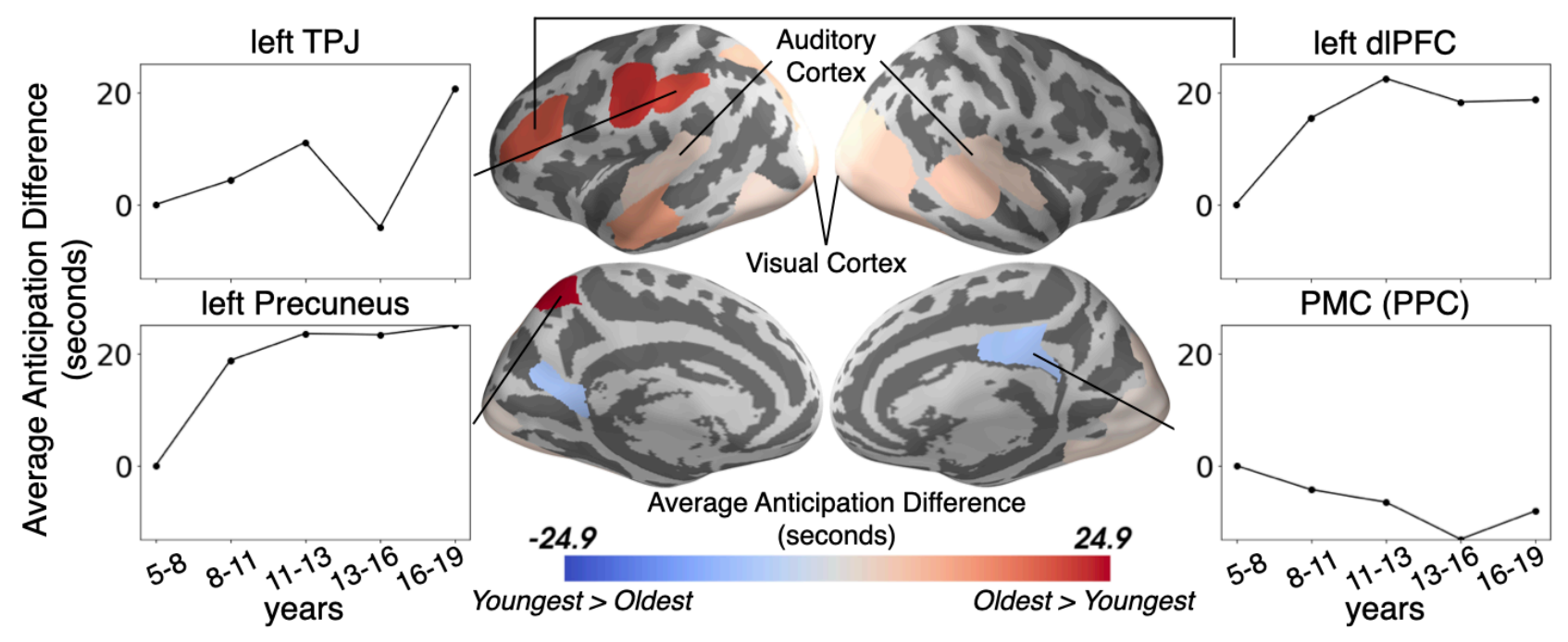

Figure 5: The timing of event boundaries shifts with age. The Oldest group represents upcoming events before the Youngest group in auditory and visual cortex, as well as left lateralized associative regions including the left temporoparietal junction, left precuneus, and left dorsolateral prefrontal cortex (reddish hues). The Youngest group anticipates events sooner in the PMC (bluish hues). Parcels shown have anticipation differences greater than age shuffled permutations $(q<0.05)$, and highlighted parcels showing age anticipation trajectories were selected post hoc for illustration.

Finally, we asked whether hippocampal responses differ as a function of age at event boundaries. We found a robust response to event boundaries in all ages (all p's $<0.0001$ for each age group's response at time 0 , see Figure $6 \mathrm{a}$, right side). This response decreases with age between 5 and 19 years old $\left(\mathrm{r}=0 .-16, \mathrm{p}=1 \times 10^{-3}, \mathrm{~N}=415\right.$; Figure 6a). As previous research has indicated that the anterior and posterior HPC may potentially respond differently to event boundaries (Reagh et al., 2020), we examined whether the anterior or posterior HPC were driving this change in boundary-driven response with age. We found that there is a significant decrease in the anterior hippocampal (aHPC) response to event boundaries with age $\left(r=-0.18, p=2 \times 10^{-4}\right.$, Figure $\left.6 \mathrm{~b}\right)$. We did not find a significant decrease in the posterior HPC (pHPC; $\mathrm{r}=-0.09, \mathrm{p}=0.07$, Figure 6c), nor a significant interaction between HPC subregions $(p=0.7)$. In none of these regions was the change in boundary-related response explained by anatomical volume changes (see Methods). 

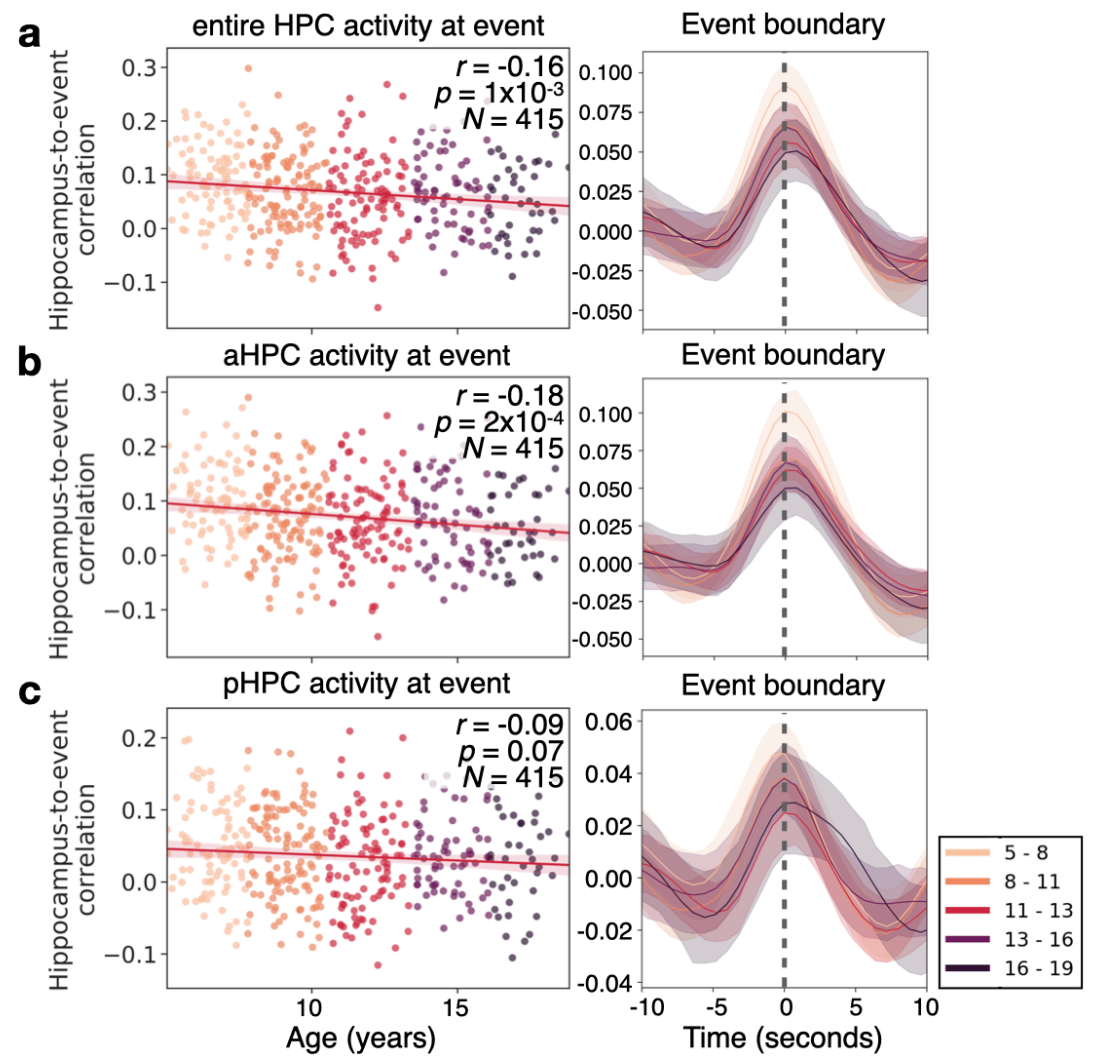

Figure 6: The hippocampus in children as young as 5 responds to event boundaries, an effect that decreases with age. (a) The event-boundary driven response decreases as a function of age in the hippocampus (HPC). This can be shown across individuals at the time point where hippocampus-to-event correlation is maximal (left), and throughout the time-course of the HPC's correlation with event boundaries. (b) The correlation between the HPC and event boundaries significantly decreases with age in the anterior HPC (aHPC), but in (c) posterior HPC ( $\mathrm{pHPC}$ ) there is no significant relationship between the boundary-driven signal and age. 


\section{Discussion}

Using a novel measure of response similarity, we found that the processes used to perceive a dynamic narrative change from ages 5 to 19 throughout the majority of the neocortex and in the hippocampus. These developmental changes are characterized by increasingly eventstructured dynamics in the neocortex, with periods of stability punctuated by rapid transitions, and anticipatory shifts in event timing. In parallel, hippocampal responses at event boundaries decrease in magnitude with age. These changes suggest that the brain's strategy for narrative processing shifts from encoding each event as a novel episode in the hippocampus to activating and maintaining generalized event knowledge in the cortex, storing only episode-specific information into long-term memory.

Older ages exhibit more across-subject synchrony in visual and auditory cortices (Figure 1), consistent with prior work showing developmental increases in ISC evoked by other similarly highly-produced animated movies (Moraczewski et al., 2018). These results are likely related to more strongly correlated semantic processing in adolescents, resulting in top-down increases in synchronous eye movements and auditory understanding (Franchak et al., 2016; Kirkorian, et al., 2012). The growth of coordinated responses may also drive the stronger event models in these regions (Figure 4). The $\mathrm{ISC}_{\mathrm{b}}$ analysis (Figure 2) found relatively small but significant shifts in the group-level responses in these regions, and auditory responses shifted slightly earlier in time with age (Figure 5), indicating that the age-related changes in sensory regions are not only largely characterized by increasing synchrony across subjects, but also (to some extent) a shift from a more child-like to more adult-like timecourse of responses.

Theory of mind regions responsible for representing the mental states of others (Gallagher, et al., 2000; Saxe, \& Kanwisher, 2003), including TPJ (Samson, et al., 2004), develop immensely in the age range studied (Sebastian, et al., 2012). We found very large age-related changes in the functional responses of TPJ (Figure 2), which included increased structuring into discrete events (Figure 4) and anticipation of upcoming events (Figure 5). Older children are likely able to construct better internal models of social and emotional structure (Dumontheil, Apperly, \& Blakemore, 2010; Pavias, et al. 2016), allowing them to form more cohesive event models. Previous work found that responses in a network of regions including TPJ shift approximately 2 seconds earlier when 6-7 year-olds watch a movie for a second time (Richardson and Saxe, 2019), but we found that 16-19 year-olds can in fact anticipate upcoming events on the first viewing of a movie clip, and that this anticipation can be as large as 20 seconds ahead of that for 5-8 year-olds. This reveals a protracted development in this region, likely due to continued growth in the ability to proactively represent the minds of others (Dumontheil, Apperly, \& Blakemore, 2010; Pavias, et al. 2016), or possibly due to a growth in attentional abilities in this region more generally (Corbetta, \& Shulman, 2002; Downar, et al., 2001).

We also found age-related shifts in dlPFC (Figure 2), as well as increasing anticipation from ages 5 to 11 (Figure 5). This region, along with other regions showing age-related shifts such as the inferior temporal cortex and orbitofrontal cortex, are a part of a network responsible for processing conceptual knowledge about the world (Binder et al., 2009). The age-driven differences in story responses may therefore be partially due to changes in the understanding of the people, actions, and culture presented in the movie (Binder et al., 2009). The dlPFC is also critical for working memory, or the ability to keep recently learned 
information readily accessible (Curtis, \& D'Esposito, 2003). The dlPFC exhibits an improvement in event structure with age (Figure 4), which may be due to the ongoing development of working memory (Kwon, Reiss, \& Menon, V., 2002), a cognitive function necessary to chunk incoming information into discrete events (Clewett, DuBrow, \& Davachi, 2019). The changes in dlPFC may also be related to development of theory of mind as discussed above, as it overlaps with the theory of mind network identified by Richardson and Saxe (2019).

The PMC, including both the PCC and RSC, was one of the few areas in which response reliability decreased with age (Figure 1), event model fits worsened (Figure 4), and anticipation decreased (Figure 5). One possibility is that PMC performs additional processing in the absence of strong schemas about an observed event; Keidel et al. (2018) found that the PMC (RSC) had significantly more activity in response to movies when the narrative topic was new than when it was a continuation of themes from the previous clip. Younger children may therefore more consistently rely on this region to understand a relatively more novel environment. This interpretation is in line with the idea that the PMC, a region that encodes information over long timescales, may help younger viewers bridge information across events (Hasson et al., 2015). The decrease in cohesive processing here may also be related to an age-driven increase in the diversity of story interpretations (Petroni et al., 2018; Szaflarski, et al., 2012).

It is of note that neither strong event representations nor an age-related change in those representations were found in the medial prefrontal cortex. This is surprising given that this region is implicated when a video is consistent with previously learned schematic knowledge (Baldassano et al., 2018; Van Kesteren et al., 2010), which we expect to be stronger in older children. However, overall ISC was low in this region, either due to idiosyncratic cognition, inconsistent neuroanatomy across subjects, or noisy signals in this region (Supplementary Figure 1), making it challenging to study this region using the group-level approaches in this study. Furthermore, as has been found previously, it may be that only a subregion of the medial prefrontal cortex represents events (Baldassano et al., 2017; Brod et al., 2017; Van Kesteren et al., 2010).

The HPC was found to reliably respond to the boundaries between events (Figure 6). This result extends evidence that the HPC tracks event changes in adults to children as young as five (Ben-Yakov and Henson, 2018; Baldassano et al., 2017; Reagh et al., 2020). This boundary-triggered response decreased with age across our sample, which is surprising given that adolescents have stronger event models and event anticipation in most of the cortical regions with significant effects. The decrease in HPC responsiveness with age may be a signature of age-related increases in the reliance on schematic representations of the environment which are stored in the cortex, thus decreasing the reliance on the HPC to encode newly encountered events (Sekeres, Winocur, \& Moscovitch, 2018). The HPC may thus have a more robust signal in young children as they use it to extract commonalities across experiences (Keresztes, et al., 2018).

Interestingly, previous research in older adults also found a decrease in the HPC response to event boundaries (Reagh et al., 2020). However, in this previous research the decrease with age was seen in the pHPC (Reagh et al., 2020). Here we find that only the response in the aHPC significantly decreases with age. This shift in age-related decline may be due to the developmental changes in the mnemonic roles along the long axis of the 
HPC (DeMaster and Ghetti, 2013; Langnes et al., 2019). The decline in the response of the aHPC may also be related to the process of semanticization (Sekeres, Winocur, \& Moscovitch, 2018), whereas older ages may be experiencing age-related mnemonic, and thus perceptual declines (Reagh et al., 2020).

Jointly fitting an HMM across children of different ages was sensible because we found that the optimal event timescales in all regions with high model fits were similar between the Youngest and Oldest groups. Children therefore develop enough narrative comprehension to segment the story in an adult-like manner by age five, though we cannot confirm whether they would be able to behaviorally segment the narrative in an adult-like way (Newtson, 1976; Newtson et al., 1977), because neither behavioral segmentation or memory data was not collected. We would expect that the largely better event model fits seen in older adolescents would also be associated with more consistent behavioral event segmentation across subjects.

Although we largely attribute the better segmentation ability and prediction to adolescents' greater experience with the world in general, it is possible that our Oldest cohort also had more previous experience watching the specific stimulus used (a clip from "Despicable Me"), as it was first released when they were young children themselves. Alternatively, younger children may have had more recent exposure to the film because it was more age appropriate for them, and this may have elicited a retrieval induced increase in HPC activity. Future research should collect data on prior stimulus exposure or aim to use stimulus-naive subjects for better control. Additionally, although we chose to use this dataset because it is one of the few large databases of movie-watching in a developmental population, the subjects recruited for this study had a high prevalence of psychopathology and limited socioeconomic diversity. Future work should generalize our findings to more diverse and representative subject samples. Finally, our analyses require stimuli that exhibit multiple transitions between events that are at least 30 seconds. We were therefore unable to make use of the other movie available in this dataset or data from the short video clips used in other developmental datasets (Alexander et al., 2017; Richardson et al., 2018). Future studies should seek to generalize these results to stimuli with greater content diversity, and duration.

Our results reveal that brain responses to a narrative movie do not simply become more synchronized across children as they age, but in fact change their dynamics and timing to become more adult-like. Brain activity patterns in many regions become more cleanly structured into discrete, consistent events, and the timing of these events shifts to anticipate upcoming content in the movie. We also found that hippocampal responses to event boundaries are present in children as young as five years old, and that these eventboundary responses are in fact larger in children than in adolescents. This study provides the groundwork for assessing how children acquire schematic knowledge about the world and ultimately deploy that knowledge at the appropriate time. 


\section{Materials and Methods}

\section{Participants}

The data in these analyses was downloaded from the Child Mind Institute's Health Brain Network (HBN) project (Alexander et al., 2017) when 1758 MRI datasets were available (data release 6). All participants provided written consent or assent, and consent was obtained from the parents or legal guardians for participants younger than 18 years. The goal of the HBN project was to collect data from subjects with heterogeneous developmental psychopathologies. As such, although the demographics and prevalence of psychopathology may not be representative of the general population, this is one of the largest developmental datasets collected while children viewed natural stimuli, and is therefore allows us to do the novel analyses outlined below. The HBN project was approved by the Chesapeake Institutional Review Board. No task functional magnetic resonance imaging (fMRI) data was available for 343 subjects. Of the remaining subjects, 803 subjects were rejected because they did not contain recordings for one of the two possible movie stimuli, they lacked one of the two possible fieldmap scans, or one of the two movies had a different number of time samples than was expected if the subject watched the entire movie. Of the remaining subjects, 183 were rejected due to unacceptably poor T1 scans, as assessed by four independent raters. Two additional subjects were eliminated as the field of view of the acquisition did not cover the whole brain, and one subject was eliminated due to a damaged $\mathrm{T} 1$ file. Four hundred and twenty six subjects remained after these eliminations. To allow for a sufficient number of subjects $(\mathrm{N}=40)$ in each age group spanning 2.76 years, with an equivalent ratio of males to females in each age group, subjects older than 19 were not used in further analyses $(\mathrm{N}=11)$. Most analyses were computed on gender-matched (22 males) samples of the Youngest (5.04 - 7.8 years, rounded in displays to 5 - 8 years) and Oldest (16.10 - 18.87 years, rounded in displays to 16 - 19 years) subjects, although illustrative analyses were also computed on the intermediate age groups (Supplementary Figure 5).

\section{MRI data collection}

Magnetic Resonance Imaging (MRI) data used in this study were collected at both the HBN Rutgers University Brain Imaging Center site on a Seimen's Trio Tim 3T scanner and the HBN CitiGroup Cornell Brain Imaging Center site on a Seimen's Prisma 3T scanner. The scan parameters at both sites were identical: $\mathrm{TR}=800 \mathrm{~ms}$, $\mathrm{TE}=30 \mathrm{~ms}$, \# slices $=60$, flip angle $=31^{\circ}$, \# volumes $=750$, voxel size $=2.4 \mathrm{~mm}$. Complete information regarding the scan parameters used for the Healthy Brain Network project can be found at: http://fcon_1000.projects.nitrc.org/indi/cmi_healthy_brain_network/MRI\%20Protocol.html.

\section{Stimulus and event rating}

fMRI data were collected while participants viewed a 10-min clip from the movie Despicable Me (01:02:09-01:12:09; presentation details available at http://fcon_1000.projects.nitrc.org/indi/cmi_healthy_brain_network/MRI\%20Protocol.html). A separate group of twenty-one adult raters (6 males) watched the clip and were given the following instructions: "The movie clip can be divided into meaningful segments. Record the times (in seconds) denoting when you feel like a meaningful segment has ended. Pause the clip at the end of the segment, write down the time in the attached spreadsheet, and provide a short, descriptive title. Try to do this when you watch the clip for the first time." 
No one reported watching the clip more than once. On average, raters found 14.4+/-6.5 events in the movie (minimum $=4$, maximum $=30$ ).

\section{Anatomical data preprocessing}

The results included in this manuscript come from preprocessing performed using fMRIPprep 1.1.4 (Esteban et al., 2018; RRID:SCR_016216), based on Nipype 1.1.1 (Gorgolewski et al., 2011; RRID:SCR_002502). The T1-weighted (T1w) image was corrected for intensity non-uniformity (INU) using 'N4BiasFieldCorrection' (Tustison et al., 2010; ANTs 2.2.0; RRID:SCR_004757), and is referred to as 'T1w-reference' throughout the workflow description. The T1w-reference was then skull-stripped using `antsBrainExtraction.sh` (ANTs 2.2.0), using OASIS as a target template. Brain surfaces were reconstructed using 'recon-all' (Dale, Fischl, and Sereno, 1999; FreeSurfer 6.0.1,RRID:SCR_001847), and the brain mask estimated was refined with a custom variation of the method used to reconcile the ANTs-derived and the FreeSurfer-derived segmentations of the cortical gray-matter in Mindboggle (Klein et al., 2017; RRID:SCR_002438). Spatial normalization to the ICBM 152 Nonlinear Asymmetrical template version 2009c (Fonov et al., 2009; MNI152NLin2009cAsym; RRID:SCR_008796) was performed through nonlinear registration with ‘antsRegistration' (ANTs 2.2.0, RRID:SCR_004757), using brain-extracted versions of both the T1w volume and the template. Brain tissue segmentation of cerebrospinal fluid (CSF), white-matter (WM) and gray-matter (GM) was performed on the brain-extracted T1w using 'fast' (Zhang, Brady, and Smith, 2001; FSL 5.0.9, RRID:SCR_002823).

\section{Functional data preprocessing}

A reference volume and its skull-stripped version were generated using custom fMRIprep methodology. For the cortical results, a B0-nonuniformity map (or fieldmap) was estimated based on two echo-planar imaging (EPI) references with opposing phase-encoding directions, with '3dQwarp' (Cox, 1996; AFNI 20160207). Based on the estimated susceptibility distortion, a corrected EPI reference was calculated for a more accurate co- $^{-}$ registration with the anatomical reference. For the hippocampal results, "Fieldmap-less" distortion correction was performed by co-registering the functional image to the samesubject T1w image with intensity inverted, (Huntenburg, 2014; Wang et al., 2017) constrained with an average fieldmap template (Treiber et al, 2016), implemented with antsRegistration (ANTs).

Head-motion parameters with respect to the BOLD reference (transformation matrices, and six corresponding rotation and translation parameters) were estimated before any spatiotemporal filtering using `mcflirt` (Jenkinson et al., 2002; FSL 5.0.9). The BOLD timeseries was resampled into its original, native space by applying a single, composite transform to correct for head-motion and susceptibility distortions. This resampled BOLD time-series will be referred to as 'preprocessed BOLD'. The BOLD reference was then co- $^{-}$ registered to the T1w reference using 'bbregister' (FreeSurfer) which implements boundary-based registration (Greve and Fischl, 2009). To account for distortions remaining in the BOLD reference, co-registration was configured with nine degrees of freedom. The BOLD time-series was resampled to the 'fsaverage6' space for the cortical analyses, and to the 'MNI152NLin2009cAsym' space for the hippocampal analyses. Confound time-series were calculated based on the preprocessed BOLD. Framewise displacement (FD) was 
calculated using the Nipype implementation (Power et al., 2013). Global signals were extracted within the CSF and the WM masks. Eight discrete cosine filters were extracted with 128 s cut-off. These nuisance regressors, as well as the head-motion estimates, were placed in a confounds file and subsequently regressed out of the BOLD time series separately for the cortex and the hippocampus using custom python scripts. All resamplings can be performed with a single interpolation step by composing all the pertinent transformations (i.e. head-motion transform matrices, susceptibility distortion correction, and co-registration to anatomical and template spaces). Gridded (volumetric) resamplings were performed using `antsApplyTransforms`(ANTs), configured with Lanczos interpolation to minimize the smoothing effects of other kernels. Non-gridded (surface) resamplings were performed using `mri_vol2surf` (FreeSurfer).

Many of the internal operations of fMRIPrep use Nilearn (version 0.4.2, Abraham et al., 2014, RRID:SCR_001362), mostly within the functional processing workflow. (For more details of the pipeline, see https://fmriprep.readthedocs.io/en/latest/workflows.html "FMRIPrep's documentation".) After these preprocessing steps were taken, the cortical surface was parcellated into the 100 parcel, seven network parcellation from Schaefer et al. (2018).

\section{Inter-subject correlation (ISC) calculations}

After preprocessing, split-half inter-subject correlation (shISC) was calculated by splitting the group within which ISC will be calculated, into two equal halves, averaging each halves' timecourse, and then correlating the two average timecourses. We chose to use shISC since we found that it could produce ISC estimates for a large group much more quickly than using pairwise ISC (pwISC) and leave-one-out ISC (looISC). Using a model similar to Nastase et al. (2019), it can be shown that, in expectation, pwISC and looISC can be calculated from shISC if the sample size is known (see Appendix B). shISC was calculated for each vertex on the cortical surface, and then averaged within each parcel.

The overall level of ISC among the participants, irrespective of age, was calculated in a randomized mixture of the Youngest and Oldest subjects (Supplementary Figure 1). This calculation can be viewed as a quality assurance step because if there is no baseline level of ISC, it is possible that the movie was not engaging to subjects, or that the sample is dominated by movement artifacts (Cohen et al., 2017; Vanderwall et al., 2020). After establishing the level of the overall group ISC, ISC was calculated within the Youngest and Oldest groups and compared between the groups, following the methods used in previous research (Cantlon and Li, 2013; Moraczewski et al., 2018).

In addition to measuring within-group ISC, we sought to compare the similarity between age groups (while controlling for within-group consistency). In order to assess a potential difference in stimulus response timecourses between the groups, while accounting for the relative level of within group correlation, we introduce a measure named betweengroup ISC $\left(\mathrm{ISC}_{\mathrm{b}}\right)$. Previous researchers have measured the timecourse correlations between subjects in two different groups (Cantlon and Li, 2013; Hasson, et al., 2009; Moraczewski et al., 2018; Nastase et al., 2019), but this measure mixes information about across-group differences and within-group consistency. For example, if two groups are identical, we would expect the between-group correlation to be the same as the within-group correlation. 
$\mathrm{ISC}_{\mathrm{b}}$ disentangles these effects, producing an estimate of the correlation between the average timecourses of two groups in the limit of infinite data (in which the group-average timecourses are measured without noise). Mathematically, ISCb is computed as the correlation between two different groups divided by the geometric mean of the correlation within each group. The denominator means that $\mathrm{ISC}_{\mathrm{b}}$ accounts for differences in ISC magnitude between the groups (see Appendix A for mathematical derivation). ISC $\mathrm{C}_{\mathrm{b}}$ was calculated between the Youngest and Oldest groups, using the shISC formulation (Appendix A).

\section{Calculating the number of events in each age group}

To determine whether the Oldest and Youngest groups perceived the same number of events in the movie, following Baldassano et al. (2017), we define an event as a stable spatial pattern of neural activity within a parcel. In each age group, and each parcel, event patterns and the number of events are chosen using a hidden markov model (HMM). To find the best fitting model, HMMs that used between 2 and 50 events (approximately logarithmically spaced) were trained and tested using five-fold cross-validation by first averaging the brain activity of four-fifths of the subjects in an age group, and then testing on the average brain activity of the held-out subjects. The number of events in an age group were defined as the average of the number of events that maximized the log likelihood of the HMM (model fit) in the held out subjects in each of the five cross-validated folds.

Although most cortical regions are expected to exhibit event structure in response to a movie stimulus, some regions will not have activity that can be well modeled by a model of events. The difference in model fit between a baseline HMM in which two events were fit and the HMM with the number of events yielding the best fit was used as a measure of the extent to which event structure was successfully detected in this region (Supplementary Figure 2). Regions that do not show substantial improvement in model fit over the twoevent model either have preferred event lengths on the scale of 300 seconds or longer (since the stimulus was 600 seconds long) or do not have event-structured responses. In 30 parcels both the Youngest and Oldest groups had a model fit difference less than 0.002 and were therefore not considered in future analyses (Supplementary Figure 2). In the remaining 70 parcels, there was no significant difference in the number of events between the groups (Supplementary Figure 3; see Statistical Significance section for permutation test details).

\section{Comparison of event boundaries in brain regions to annotations}

Following the methods of Lee et al. (2021), we jointly fit an HMM in each parcel to the Youngest and Oldest groups simultaneously. This joint-fitting procedure assumes that both groups share the same number of events and brain pattern in each event. Both the degree of model fit and the timing of these events can vary between the groups. The number of events and event patterns were determined by finding the maximum log likelihood of the model-fit on the average of five held-out folds of the data from all five age groups (including ages in between the Youngest and Oldest). This fitting procedure is similar to the case where HMMs are fit separately in each age group, except in this case, the log likelihood of the model-fit is averaged over all age groups in addition to all training folds.

We next evaluated the correspondence between the HMM-derived event boundaries and boundaries from human raters. To determine where event transitions occur in the model, 
we computed the derivative of the expected event label over time for each age group and parcel. The behavioral boundaries were derived from the number of boundary annotations at each timepoint convolved with a canonical hemodynamic response function (HRF, one parameter gamma model from AFNI's 3dDeconvolve; Lee et al., 2021). We related the two timecourses using Pearson correlation (Supplementary Figure 4).

\section{Determining the model fit in each age group}

The log likelihood, or model-fit, of the best-fitting jointly-fit HMM was compared between the Oldest and Youngest groups. For illustration of the change in log likelihood with age, the average log likelihood was also computed on similarly sized folds of the age groups in between the Youngest and Oldest groups.

\section{Measuring changes in event timing across age}

Following the procedure from Lee et al. (2021), using the jointly-fit HMMs described in the previous section, the probability that each fold of the held-out data was in any of the events was determined for each age group in each parcel. Averaging these probabilities across all five-folds yielded a time-point by number of events matrix. We then computed (separately for each group) the expected value of the event present at each timepoint. Summing over these expectation values produced a value describing the amount of time that this region spent in earlier versus later events, with larger numbers indicating that more time was spent in later events. If the sum of these expectation values is greater in one age group than in the other, this indicates that this age group represents upcoming events before the other age group.

\section{Statistical Significance}

To test for a significant difference between the Youngest and Oldest groups in all of the above analyses, Age permuted values were computed for each parcel for the difference in ISC magnitude (absolute value of ISC difference), ISC $\mathrm{C}_{b}$, number of events in age group specific HMMs, difference in model fit (maximum log likelihood) for jointly-fit HMMs, and difference in event timing for jointly-fit HMMs. All age permuted groups had the same gender distribution as the true sample. For each parcel, the calculation of the real (nonpermuted) value, as well as all permuted values were calculated in five random subsamples of each age group (Youngest and Oldest) since random subsampling generates slightly different results. Both the real and permuted values were then averaged across all five subsamples to calculate both the values reported here and their significance. The permuted group assignment in each permutation was therefore maintained across the five subsamples.

If in any parcel, for any test, the number of the permuted values greater than (or less than, in the case of $\mathrm{ISC}_{\mathrm{b}}$ ) the true value was less than $5 \%$ in any of the five random subsamples after 100 permutations, 1000 additional permutations were run. 1000 additional permutations were computed until every parcel had at least one permuted value greater than (or less than) the value in the original data, or 6000 permutations had been computed (whichever came first). If after 6000 permutations, there were still no permuted values greater than (or less than) the value in the original data, the original data was included as a permuted value for significance tests. 
All HMMs were initially trained on the original (non age-permuted) training data (fourfifths of the full dataset). To compute permuted differences of the number of events, agespecific HMMs were tested on age-permuted testing data for all numbers of events. The jointly-fit HMMs were tested on permuted data for only the best fitting number of events, determined from the original data. In all statistical tests, the false discovery rate (FDR) of parcel differences was controlled for by setting the expected proportion of false positives to .05 (Benjamini and Hochberg, 1995). The code for all the included analyses is available at: https://github.com/samsydco/HBN

\section{Hippocampal segmentation and event rating correlation}

The hippocampus was defined using the Freesurfer segmentation generated using fMRIPrep 1.5.6 (Esteban et al., 2018). Consistent with previous studies, the anterior hippocampus (aHPC) was defined as voxels anterior to $\mathrm{y}=-21 \mathrm{~mm}$ in MNI space, and the posterior hippocampus ( $\mathrm{pHPC}$ ) was defined as voxels including and posterior to $\mathrm{y}=-21 \mathrm{~mm}$ in MNI space (Poppenk et al., 2013; Zeidman et al. 2015). For each subject, voxel-wise activity was averaged within the entire hippocampus, the aHPC, and the pHPC. Each subject's hippocampal timecourses were then correlated with an event boundary timecourse obtained behaviorally from raters. Similarly to the behavioral timecourse related to the HMM, here, the event boundary timecourse was computed by convolving the number of boundary annotations at each timepoint during the movie with a canonical HRF (Lee et al., 2021). The hippocampal timecourses and the event boundary timecourse were correlated at differences in delay of up to 10 seconds. These lagged correlations were computed because previous research has shown that the hippocampal response to event boundaries can follow event boundaries by several seconds (Baldassano et al., 2020; Ben-Yakov and Henson, 2018, Dubrow \& Davachi, 2016, Reagh et al., 2020). The correlation values for each hippocampal region, temporal lag, and subject were then grouped according to the age of the subject. The Pearson's correlation at lag 0 between age and the hippocampus-to-event boundary correlation was assessed across all subjects in the entire hippocampus as well as in all hippocampal subregions.

Previous research has shown that the volume of the hippocampus may decrease with age (Gogtay et al., 2006). The volume of the hippocampus and its subregions was therefore measured as the number of voxels that the Freesurfer parcellation allotted to the hippocampus. The aHPC volume was the number of voxels anterior to $\mathrm{y}=-21 \mathrm{~mm}$ in $\mathrm{MNI}$ space, and the pHPC volume was the number of voxels including and posterior to $\mathrm{y}=-21$ $\mathrm{mm}$ in MNI space. The volume of the entire hippocampus and aHPC correlated with subject age (entire HPC: $\mathrm{r}=-0.1, \mathrm{p}=0.03$, aHPC: $\mathrm{r}=-0.1, \mathrm{p}=0.03$ ) while the volume of the $\mathrm{pHPC}$ $\operatorname{did}$ not $(\mathrm{r}=-0.05, \mathrm{p}=0.3)$. However, in none of the regions was the event boundary response significantly correlated with the change in volume (entire $\mathrm{HPC}, \mathrm{r}=0.01, \mathrm{p}=0.9$, aHPC , $\mathrm{r}=-0.01, \mathrm{p}=0.9$, $\mathrm{pHPC}, \mathrm{r}=-0.03, \mathrm{p}=0.5$ ).

\section{Acknowledgements}

We thank Andrew Africk for providing funding support for S.C., Michael Chow for helping to formulate the math for $\mathrm{ISC}_{b}$, and Nora Newcombe, Paul A. Bloom, Rolando MasisObando, and Vishnu "Deepu" Murty for their edits and suggestions. 
bioRxiv preprint doi: https://doi.org/10.1101/2021.04.12.439526; this version posted April 14, 2021. The copyright holder for this preprint (which was not certified by peer review) is the author/funder, who has granted bioRxiv a license to display the preprint in perpetuity. It is made available under aCC-BY 4.0 International license.

\section{Competing Interests}

The authors have no competeing interests to declare. 


\section{References}

Abraham A, Pedregosa F, Eickenberg M, Gervais P, Mueller A, Kossaifi J, Gramfort A, Thirion B, Varoquaux G. Machine learning for neuroimaging with scikit-learn. Front in Neuroinf 8:14. 2014. doi:10.3389/fninf.2014.00014.

Alexander, L. M., Escalera, J., Ai, L., Andreotti, C., Febre, K., Mangone, A., ... \& Milham, M. P. (2017). An open resource for transdiagnostic research in pediatric mental health and learning disorders. Scientific data, 4(1), 1-26.

Aslin, R. N., \& Smith, L. B. (1988). Perceptual development. Annual review of psychology, 39(1), 435-473.

Baldassano, C., Chen, J., Zadbood, A., Pillow, J. W., Hasson, U., \& Norman, K. A. (2017). Discovering event structure in continuous narrative perception and memory. Neuron, 95(3), 709-721.

Baldassano, C., Hasson, U., \& Norman, K. A. (2018). Representation of real-world event schemas during narrative perception. Journal of Neuroscience, 38(45), 9689-9699.

Ben-Yakov, A., \& Henson, R. N. (2018). The hippocampal film editor: Sensitivity and specificity to event boundaries in continuous experience. Journal of Neuroscience, 38(47), 10057-10068.

Benjamini, Y., Hochberg, Y. (1995). Controlling the false discovery rate: a practical and powerful approach to multiple testing. Journal of the Royal Statistical Society: Series B (Methodological), 57(1), 289-300.

Binder, J. R., Desai, R. H., Graves, W. W., \& Conant, L. L. (2009). Where is the semantic system? A critical review and meta-analysis of 120 functional neuroimaging studies. Cerebral cortex, 19(12), 2767-2796.

Binder, J. R., \& Desai, R. H. (2011). The neurobiology of semantic memory. Trends in cognitive sciences, 15(11), 527-536.

Brod, G., Lindenberger, U., \& Shing, Y. L. (2017). Neural activation patterns during retrieval of schema-related memories: Differences and commonalities between children and adults. Developmental science, 20(6), e12475.

Cantlon, J.F., Li, R. (2013). Neural activity during natural viewing of sesame street statistically predicts test scores in early childhood. PLoS Biology, 11(1), e1001462.

Carpendale, J., \& Lewis, C. (2006). How children develop social understanding. Blackwell Publishing.

Casillas, M., \& Frank, M. C. (2017). The development of children's ability to track and predict turn structure in conversation. Journal of memory and language, 92, 234-253. 
Chen, J., Leong, Y. C., Honey, C. J., Yong, C. H., Norman, K. A., \& Hasson, U. (2017). Shared memories reveal shared structure in neural activity across individuals. Nature neuroscience, 20(1), 115-125.

Chi, M.T.H. (1978). Knowledge structures and memory development. In R. Siegler (Ed.), Children's thinking: What develops? (pp. 73-96). Hillsdale, NJ: Erlbaum.

Clark, E. V. (1973). What's in a word? On the child's acquisition of semantics in his first language. In T. E. Moore (Ed.), Cognitive development and the acquisition of language (pp. 65-110). New York, NY: Academic Press.

Clewett, D., DuBrow, S., \& Davachi, L. (2019). Transcending time in the brain: How event memories are constructed from experience. Hippocampus, 29(3), 162-183.

Corbetta, M., \& Shulman, G. L. (2002). Control of goal-directed and stimulus-driven attention in the brain. Nature reviews neuroscience, 3(3), 201-215.

Cox RW. AFNI: software for analysis and visualization of functional magnetic resonance neuroimages. Comput Biomed Res. 1996 Jun;29(3):162-73. doi:10.1006/cbmr.1996.0014.

Curtis, C. E., \& D'Esposito, M. (2003). Persistent activity in the prefrontal cortex during working memory. Trends in cognitive sciences, 7(9), 415-423.

Dale A, Fischl B, Sereno MI. Cortical Surface-Based Analysis: I. Segmentation and Surface Reconstruction. Neuroimage. 1999;9(2):179-94. doi:10.1006/nimg.1998.0395.

DeMaster, D. M., \& Ghetti, S. (2013). Developmental differences in hippocampal and cortical contributions to episodic retrieval. Cortex, 49(6), 1482-1493.

Downar, J., Crawley, A. P., Mikulis, D. J., \& Davis, K. D. (2001). The effect of task relevance on the cortical response to changes in visual and auditory stimuli: an eventrelated fMRI study. Neuroimage, 14(6), 1256-1267.

DuBrow, S., \& Davachi, L. (2016). Temporal binding within and across events. Neurobiology of learning and memory, 134, 107-114.

Dumontheil, I., Apperly, I. A., \& Blakemore, S. J. (2010). Online usage of theory of mind continues to develop in late adolescence. Developmental science, 13(2), 331-338.

Esteban O, Markiewicz CJ, Blair RW, Moodie CA, Isik AI, Erramuzpe A, Kent JD, Goncalves M, DuPre E, Snyder M, Oya H, Ghosh SS, Wright J, Durnez J, Poldrack RA, Gorgolewski KJ. fMRIPrep: a robust preprocessing pipeline for functional MRI. Nat Meth. 2018; doi:10.1038/s41592-018-0235-4

Fonov VS, Evans AC, McKinstry RC, Almli CR, Collins DL. Unbiased nonlinear average age-appropriate brain templates from birth to adulthood. NeuroImage; Amsterdam. 2009 Jul 1;47:S102. doi:10.1016/S1053-8119(09)70884-5. 
Franchak, J. M., Heeger, D. J., Hasson, U., \& Adolph, K. E. (2016). Free viewing gaze behavior in infants and adults. Infancy, 21(3), 262-287.

Gallagher, H. L., Happé, F., Brunswick, N., Fletcher, P. C., Frith, U., \& Frith, C. D. (2000). Reading the mind in cartoons and stories: an fMRI study of 'theory of mind'in verbal and nonverbal tasks. Neuropsychologia, 38(1), 11-21.

Geerligs, L., van Gerven, M., Campbell, K., \& Güçlü, U. (2021). A nested cortical hierarchy of neural states underlies event segmentation in the human brain. bioRxiv.

Gogtay, N., Nugent III, T. F., Herman, D. H., Ordonez, A., Greenstein, D., Hayashi, K. M., ... \& Thompson, P. M. (2006). Dynamic mapping of normal human hippocampal development. Hippocampus, 16(8), 664-672.

Gorgolewski K, Burns CD, Madison C, Clark D, Halchenko YO, Waskom ML, Ghosh SS. Nipype: a flexible, lightweight and extensible neuroimaging data processing framework in python. Front Neuroinform. 2011 Aug 22;5(August):13. doi:10.3389/fninf.2011.00013.

Grady, C. (2012). The cognitive neuroscience of ageing. Nature Reviews Neuroscience, 13(7), 491-505.

Greve DN, Fischl B. Accurate and robust brain image alignment using boundary-based registration. Neuroimage. 2009 Oct;48(1):63-72. doi:10.1016/j.neuroimage.2009.06.060.

Han, S., Jiang, Y., \& Humphreys, G. W. (2007). Watching cartoons activates the medial prefrontal cortex in children. Chinese Science Bulletin, 52(24), 3371-3375.

Hasson, U., Avidan, G., Gelbard, H., Vallines, I., Harel, M., Minshew, N., \& Behrmann, M. (2009). Shared and idiosyncratic cortical activation patterns in autism revealed under continuous real-life viewing conditions. Autism Research, 2(4), 220-231.

Hasson, U., Chen, J., \& Honey, C. J. (2015). Hierarchical process memory: memory as an integral component of information processing. Trends in cognitive sciences, 19(6), 304-313.

Huntenburg JM. Evaluating nonlinear coregistration of BOLD EPI and T1w images. Freie Universität Berlin; 2014. Available from: http://hdl.handle.net/11858/00-001M-0000-002B1CB5-A.

Jenkinson M, Bannister P, Brady M, Smith S. Improved optimization for the robust and accurate linear registration and motion correction of brain images. Neuroimage. 2002 Oct;17(2):825-41. doi:10.1006/nimg.2002.1132.

Keidel, J. L., Oedekoven, C. S., Tut, A. C., \& Bird, C. M. (2018). Multiscale integration of contextual information during a naturalistic task. Cerebral Cortex, 28(10), 3531-3539.

Keresztes, A., Ngo, C. T., Lindenberger, U., Werkle-Bergner, M., \& Newcombe, N. S. (2018). Hippocampal maturation drives memory from generalization to specificity. Trends in Cognitive Sciences, 22(8), 676-686. 
Kirkorian, H. L., Anderson, D. R., \& Keen, R. (2012). Age differences in online processing of video: An eye movement study. Child development, 83(2), 497-507.

Klein A, Ghosh SS, Bao FS, Giard J, Häme Y, Stavsky E, et al. Mindboggling morphometry of human brains. PLoS Comput Biol 13(2): e1005350. 2017. doi:10.1371/journal.pcbi.1005350.

Kwon, H., Reiss, A. L., \& Menon, V. (2002). Neural basis of protracted developmental changes in visuo-spatial working memory. Proceedings of the National Academy of Sciences, 99(20), 13336-13341.

Langnes, E., Vidal-Piñeiro, D., Sneve, M. H., Amlien, I. K., Walhovd, K. B., \& Fjell, A. M. (2019). Development and decline of the hippocampal long-axis specialization and differentiation during encoding and retrieval of episodic memories. Cerebral Cortex, 29(8), 3398-3414.

Lee, C., Aly, M., and Baldassano, C. (2021). Anticipation of temporally structured events in the brain. bioRxiv.

Lerner, Y., Scherf, K. S., Katkov, M., Hasson, U., \& Behrmann, M. (2019). Age-Related Changes in Neural Networks Supporting Complex Visual and Social Processing in Adolescence. bioRxiv, 650887.

Moraczewski, D., Chen, G., \& Redcay, E. (2018). Inter-subject synchrony as an index of functional specialization in early childhood. Scientific reports, 8(1), 1-12.

Moraczewski, D., Nketia, J., \& Redcay, E. (2020). Cortical temporal hierarchy is immature in middle childhood. NeuroImage, 116616.

Nastase, S. A., Gazzola, V., Hasson, U., \& Keysers, C. (2019). Measuring shared responses across subjects using intersubject correlation.

Nelson, K. (Ed.). (1986). Event knowledge structure and function in development. Erlbaum.

Newtson, D. (1976). Foundations of attribution: The perception of ongoing behavior. New directions in attribution research, 1, 223-247.

Newtson, D., Engquist, G. A., \& Bois, J. (1977). The objective basis of behavior units. Journal of Personality and social psychology, 35(12), 847.

Pavias, M., van den Broek, P., Hickendorff, M., Beker, K., \& Van Leijenhorst, L. (2016). Effects of social-cognitive processing demands and structural importance on narrative recall: Differences between children, adolescents, and adults. Discourse Processes, 53(5-6), 488-512.

Petroni, A., Cohen, S. S., Ai, L., Langer, N., Henin, S., Vanderwal, T., ... \& Parra, L. C. (2018). The variability of neural responses to naturalistic videos change with age and sex. Eneuro, 5(1). 
Poldrack, R. A., Baker, C. I., Durnez, J., Gorgolewski, K. J., Matthews, P. M., Munafò, M. R., ... \& Yarkoni, T. (2017). Scanning the horizon: towards transparent and reproducible neuroimaging research. Nature reviews neuroscience, 18(2), 115.

Poppenk, J., Evensmoen, H. R., Moscovitch, M., \& Nadel, L. (2013). Long-axis specialization of the human hippocampus. Trends in cognitive sciences, 17(5), 230-240.

Power JD, Mitra A, Laumann TO, Snyder AZ, Schlaggar BL, Petersen SE. Methods to detect, characterize, and remove motion artifact in resting state fMRI. Neuroimage. 2013 Aug 29;84:320-41. doi:10.1016/j.neuroimage.2013.08.048.

Raichle, M. E., MacLeod, A. M., Snyder, A. Z., Powers, W. J., Gusnard, D. A., \& Shulman, G. L. (2001). A default mode of brain function. Proceedings of the National Academy of Sciences, 98(2), 676-682.

Ralph, M. A. L., Jefferies, E., Patterson, K., \& Rogers, T. T. (2017). The neural and computational bases of semantic cognition. Nature Reviews Neuroscience, 18(1), 42-55.

Reagh, Z. M., Delarazan, A. I., Garber, A., \& Ranganath, C. (2020). Aging alters neural activity at event boundaries in the hippocampus and Posterior Medial network. Nature communications, 11(1), 1-12.

Richardson, H., \& Saxe, R. (2020). Development of predictive responses in theory of mind brain regions. Developmental science, 23(1), e12863.

Richardson, H., Lisandrelli, G., Riobueno-Naylor, A., \& Saxe, R. (2018). Development of the social brain from age three to twelve years. Nature communications, 9(1), 1-12.

Saxe, R., \& Kanwisher, N. (2003). People thinking about thinking people: the role of the temporo-parietal junction in "theory of mind". Neuroimage, 19(4), 1835-1842.

Schaefer A, Kong R, Gordon EM, Laumann TO, Zuo XN, Holmes AJ, Eickhoff SB, Yeo BTT. Local-Global parcellation of the human cerebral cortex from intrinsic functional connectivity MRI. Cerebral Cortex, 29:3095-3114, 2018

Schneider, W., \& Pressley, M. (2013). Memory development between two and twenty. Psychology Press.

Sebastian, C. L., Fontaine, N. M., Bird, G., Blakemore, S. J., De Brito, S. A., McCrory, E. J., \& Viding, E. (2012). Neural processing associated with cognitive and affective Theory of Mind in adolescents and adults. Social cognitive and affective neuroscience, 7(1), 53-63.

Sekeres, M. J., Winocur, G., \& Moscovitch, M. (2018). The hippocampus and related neocortical structures in memory transformation. Neuroscience letters, 680, 39-53.

Shaw, P., Kabani, N. J., Lerch, J. P., Eckstrand, K., Lenroot, R., Gogtay, N., ... \& Wise, S. P. (2008). Neurodevelopmental trajectories of the human cerebral cortex. Journal of neuroscience, 28(14), 3586-3594. 
Supekar, K., Uddin, L. Q., Prater, K., Amin, H., Greicius, M. D., \& Menon, V. (2010). Development of functional and structural connectivity within the default mode network in young children. Neuroimage, 52(1), 290-301.

Szaflarski, J. P., Altaye, M., Rajagopal, A., Eaton, K., Meng, X., Plante, E., \& Holland, S. K. (2012). A 10-year longitudinal fMRI study of narrative comprehension in children and adolescents. Neuroimage, 63(3), 1188-1195.

Treiber JM, White NS, Steed TC, Bartsch H, Holland D, Farid N, McDonald CR, Carter BS, Dale AM, Chen CC. Characterization and Correction of Geometric Distortions in 814 Diffusion Weighted Images. PLoS One. 2016 Mar 30;11(3):e0152472. doi:10.1371/journal.pone.0152472.

Tustison NJ, Avants BB, Cook PA, Zheng Y, Egan A, Yushkevich PA, Gee JC. N4ITK: improved N3 bias correction. IEEE Trans Med Imaging. 2010 Jun;29(6):1310-20. doi:10.1109/TMI.2010.2046908.

Van Kesteren, M. T., Fernández, G., Norris, D. G., \& Hermans, E. J. (2010). Persistent schema-dependent hippocampal-neocortical connectivity during memory encoding and postencoding rest in humans. Proceedings of the National Academy of Sciences, 107(16), $7550-7555$.

Vanderwal, T., Eilbott, J., \& Castellanos, F. X. (2019). Movies in the magnet: Naturalistic paradigms in developmental functional neuroimaging. Developmental cognitive neuroscience, 36, 100600.

Wang S, Peterson DJ, Gatenby JC, Li W, Grabowski TJ, Madhyastha TM. Evaluation of Field Map and Nonlinear Registration Methods for Correction of Susceptibility Artifacts in Diffusion MRI. Front Neuroinform. $2017 \quad$ [cited 2017 Feb 21];11. doi:10.3389/fninf.2017.00017.

Zeidman, P., Mullally, S. L., \& Maguire, E. A. (2015). Constructing, perceiving, and maintaining scenes: hippocampal activity and connectivity. Cerebral Cortex, 25(10), 38363855 .

Zhang Y, Brady M, Smith S. Segmentation of brain MR images through a hidden Markov random field model and the expectation-maximization algorithm. IEEE Trans Med Imaging. 2001 Jan;20(1):45-57. doi:10.1109/42.906424. 